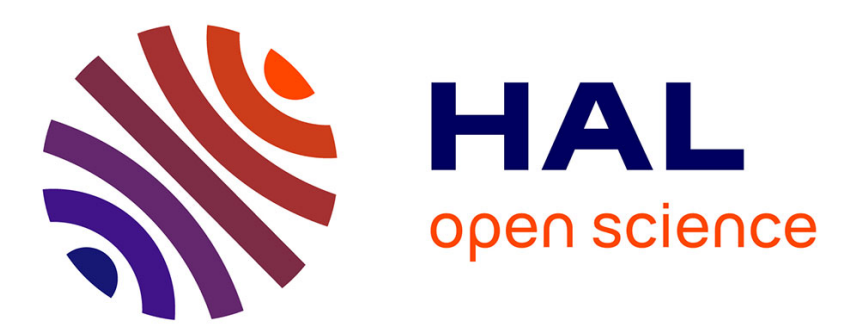

\title{
First Principles Investigation of the Spectral Properties of Neutral, Zwitterionic, and bis-Cationic Azaacenes
}

Patricia Guevara-Level, Simon Pascal, Olivier Siri, Denis Jacquemin

\section{To cite this version:}

Patricia Guevara-Level, Simon Pascal, Olivier Siri, Denis Jacquemin. First Principles Investigation of the Spectral Properties of Neutral, Zwitterionic, and bis-Cationic Azaacenes. Physical Chemistry Chemical Physics, 2019, 10.1039/C9CP04835A . hal-02304187

\section{HAL Id: hal-02304187 \\ https://hal.science/hal-02304187}

Submitted on 31 Jan 2020

HAL is a multi-disciplinary open access archive for the deposit and dissemination of scientific research documents, whether they are published or not. The documents may come from teaching and research institutions in France or abroad, or from public or private research centers.
L'archive ouverte pluridisciplinaire HAL, est destinée au dépôt et à la diffusion de documents scientifiques de niveau recherche, publiés ou non, émanant des établissements d'enseignement et de recherche français ou étrangers, des laboratoires publics ou privés. 


\title{
First Principles Investigation of the Spectral Prop- erties of Neutral, Zwitterionic, and bis-Cationic Azaacenes. $^{\dagger}$
}

\author{
Patricia Guevara Level, ${ }^{a}$ Simon Pascal, ${ }^{b}$ Olivier Siri ${ }^{* a}$ and Denis Jacquemin ${ }^{* a}$
}

Received Date

Accepted Date

DOI: $10.1039 / x x x x x x x x x x$

www.rsc.org/journalname

\begin{abstract}
An in-depth investigation of the optical properties of recently-synthesized linear azaacene derivatives of various electronic nature (neutral, dicationic, and zwitterionic) is presented. Our simulations include not only the determination of the vertical transitions, but also of the adiabatic energies and vibrationally-resolved spectra using the Adiabatic Hessian approach in connection with the Time-Dependent Density Functional Theory (TD-DFT) and second-order Coupled-Cluster (CC2) methods. We show that the theoretical results are in excellent agreement with experiment for both the $\lambda_{\max }$ and the band topologies, a statement holding in the full visible domain. We also analyse the nature of the key vibrations explaining the specific band shapes of these compounds. In addition, we investigate a series of yet-unknown substituted systems in an effort to design new compounds with improved (redshifted) properties for optoelectronics applications.
\end{abstract}

\section{Introduction}

Pentacene can be viewed as the reference compound in organic electronics because of its high intrinsic mobility of charges. 12 However, this molecule suffers from a long-term stability mainly due to photooxidation processes which limit its use in wide range of applications. To overcome this drawback, one can envisage to introduce nitrogen atoms which offers a number of opportunities to manipulate and control the electronic properties, the stability, and the supramolecular arrangement in the solid state. 10 In this context, the nitrogenated isostructural derivatives of pentacene (fused-azaacenes) are attractive building blocks for developing a wide array of optical and electrical applications. Indeed, phototransistors, solar cells, photoelectrochemical cells, organic light-emitting diodes (OLEDs), sensors, and memory devices based on these molecular skeletons have appeared during the last decade.11-16 Analogous zwitterionic azaacenes have received also a significant attention as the presence of both positive and negative charges within a single conjugated system often gives rise to properties not otherwise accessible. In this specific field, one should certainly mention the numerous key contributions of the Koutentis group, who synthesized and characterized many compounds of this family. $\frac{17-19}{10}$ In one of their most recent investigations, 18 they proposed the molecules sketched in Figure 1. More specifically, they developed a new synthetic route for reaching dyes $\mathbf{1}$ and $\mathbf{2}$ and also obtained UV-vis, NMR,

$0^{a}$ Laboratoire CEISAM -UMR CNRS 6230,Université de Nantes, 2 Rue de la Houssinière, BP 92208, 44322 Nantes Cedex 3, France ; Tel.: +33-2-51-12-55-64 ; E-mail: Denis.Jacquemin@univ-nantes.fr.

$0^{b}$ Aix Marseille Université, CNRS UMR 7325, CINAM, Campus de Luminy, case 913, Marseille 13288 Cedex 09, France; E-mail: olivier.siri@univ-amu.fr.

$0 \dagger$ Electronic Supplementary Information (ESI) available: i) Cartesian coordinates; ii) Molecular orbitals; iii) List of vertical transition energies; iv) Density Difference Plots; and v) Vibrationally resolved spectra.. See DOI: 10.1039/b000000x/ and cyclic voltammograms for all compounds. The reported absorption spectra are particularly rich, with not only changes of band positions when going from one compound to another, but also a strong multi-peak structure hinting at the presence of several dipole-allowed transition to electronic excited states (ES), each accompanied by strong vibrationnal couplings. In addition to the experimental investigation, theoretical calculations were provided to support the measurements. 18 More specifically, Time-Dependent Density Functional Theory (TD-DFT) calculations with the B3LYP exchange-correlation functional have been performed to obtain vertical transition energies. 18 Although such an approach is computationally efficient and provides a qualitative characterization of the nature of the relevant ES, it suffers from two drawbacks. First, vertical transition energies do not offer well-grounded theory-measurement comparisons, because vertical values are inaccessible experimentally. To obtain theoretical data directly comparable to the measurements, one should indeed determine 0-0 energies and/or band shapes, 20 though this implies a much higher computational cost as the ES Hessian needs to be determined, or at least approximated, for each state of interest. Given the complex nature of the experimental spectra, ${ }^{18}$ such calculations might nevertheless be mandatory to properly assign all bands. Second, for cyanine derivatives, and compounds 3-5 of Figure 1 obviously present moieties belonging to this category, TD-DFT transition energies tend to be rather inaccurate $^{28-30}$ and one has often to resort to more advanced theoretical approaches, e.g., second-order coupled-cluster (CC2), to attain a satisfying accuracy. 30,32 Given these facts, we perform state-of-the-art calculations with a focus on the vibronic shapes in the present contribution.

The present study is organized as follows : we first detail the selected computational procedures, before presenting the results related to the known dyes. We focus on the absorption spectra of the compounds of Ref. 18, identifying and characterizing the 

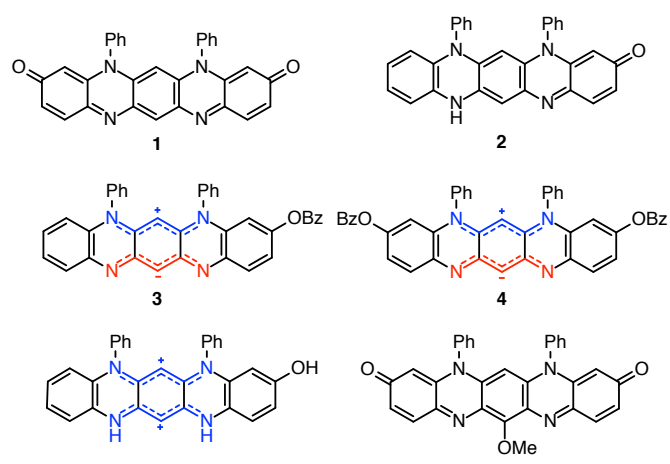

Fig. 1 Molecular structures of known dyes studied herein: $\quad 1 \quad$ (Isodiphenylfluorindone), 2 (Isodephenylfluorindinone), 3 (3-Benzoyloxyisodephenylfluorindine), 4 (3,9Dibenzoyloxyisodiphenylfluorindine), 5 (3-Hydroxy-12,14-diphenyl7,14-dihydroquinoxalino[2,3-b]phenazine-5,12-diium), and 6 (13Methoxyisodiphenylfluorindone). The positively (negatively) charged cyanine moieties are highlighted in blue (red) in dyes 3-5.

key electronic states and their vibronic couplings with the ground state. Finally in an effort to clearing the path to new interesting structures, we investigate the impact of different substitution strategies on the $\lambda_{\max }$ and band shapes.

\section{Computational Details}

DFT and TD-DFT calculations are carried out with the Gaussian 16 program package. $\frac{33}{}$ A tight convergence threshold of $1 \mathrm{x}$ $10^{-10}$ au is applied during the Self-Consistent Field (SCF) procedure, and we use the so-called ultrafine DFT integration grid [pruned $(99,590)$ grid] to obtain numerically-reliable exchangecorrelation energies. The geometries of both the ground state (GS) and several low-lying ES are optimized using PBE0, $34[35$ a global hybrid including a quarter of exact exchange and known to provide an accurate description of ES properties in dyes not undergoing strong intramolecular charge-transfer (CT). ${ }^{24 \mid 36}$ The geometry optimizations are carried out with the $6-31+\mathrm{G}(\mathrm{d})$ atomic basis set until the residual mean force is smaller than $1 \times 10^{-5}$ au (tight convergence in Gaussian). Following the optimizations, analytic frequency calculations have been performed for both the GS and ES in order to confirm the absence of imaginary frequency and to obtain the vibrational frequencies and dipole derivatives used in the vibronic calculations (see below). The transition energy calculations are determined with the same functional and the aug-cc-pVTZ basis set. During all the DFT and TD-DFT calculation steps, the solvent effects are modeled through the application of the well-known Polarizable Continuum Model (PCM). 37 Following experiment, 18 the selected solvent is dichloromethane (DCM), an aprotic medium for which PCM is an adequate model. The non-equilibrium limit of the PCM approach is used during the calculation of vertical absorption energies, as only the electrons of the media have time to react to the extremely fast change of electronic state following photon absorption. There exist several schemes allowing coupling PCM to TD-DFT,, 38 and we apply here the linear-response (LR) approach ${ }^{3940}$ that is suited for local bright transitions. In LR-PCM, the solvent effect depends on the transition dipole moment. Of course, some of the considered transitions present a partial CT character (see below) and it is also worth to evaluate the results obtained with a state-specific solvent model, that accounts for the change of total dipole mo- ment between the GS and the considered ES. 38 We have here selected the corrected LR model (cLR) of Caricato, Mennucci and coworkers, 41 to assess the magnitude of sate-specific corrections and considered the $S_{0} \rightarrow S_{1}$ and $S_{0} \rightarrow S_{4}$ excitations in 3 , the former presenting a partial CT character (see below). For the former transition, the cLR correction as compared to the gas-phase like $\omega_{0}$ value is rather small $(-0.03 \mathrm{eV})$ and it becomes totally negligible for the second transition $(0.00 \mathrm{eV})$. This is contrast to the LR solvent correction that amount to $-0.10 \mathrm{eV}$ for both excitations. This justifies the neglect of state-specific corrections in the following.

Vibrationally resolved spectra are determined within the Adiabatic Hessian approach, 4243 the most refined "classical" approximation that accounts for Duschinsky rotation and changes of normal modes between the GS and the ES. These calculations are performed using the FCclasses program 2144 on the basis of the harmonic vibrational spectra obtained with DFT and TD-DFT. The Franck-Condon (FC) approximation is employed as we consider strongly dipole allowed transition $(f>0.1)$ The reported spectra are simulated using a convoluting Gaussian function presenting a half width at half-maximum (HWHM) that is adjusted to allow direct comparisons with experiments (typical value 0.04 $\mathrm{eV}$ ). A maximum number of 25 overtones for each mode and 20 combination bands on each pair of modes are used during the FCclasses calculations. The number of integrals is set to allow proper convergence of the FC factor $(\geq 0.9)$. The adiabatic energies used during the calculations of the vibrationally resolved spectra are obtained using the same composite approach as in Ref. 45. In this protocol, the TD-DFT values are corrected using CC2 transition energies. As stated in the Introduction, applying such a second-order wavefunction approach to determine transition energies is likely mandatory here here as several compounds present a cyanine segments. We note that previous works have shown that it is however valid to use TD-DFT structures and vibrations on organic dyes, as obtaining CC2 geometries would not significantly improve the results. 46 48 The CC2 calculations are performed with the Turbomole code, 49 applying the resolutionof-identity approach, and selecting the aug-cc-pVDZ atomic basis set.

We stress that, in the body of the work, all reported $\lambda_{\max }$ are obtained through the above-mentioned procedure, i.e., these wavelengths are maxima of the vibronic progression determined using TD-DFT vibrations and CC2-corrected adiabatic energies, i.e., are not "simple" vertical values extracted from a TD-DFT calculation performed on a frozen GS geometry. To provide an idea of the impact of CC2 corrections on the adiabatic energies, it amounts to $-0.068 \mathrm{eV}$ and $-0.116 \mathrm{eV}$ for the lowest transitions in 2 and $\mathbf{3}$, respectively. One notices that the correction is larger for the zwitterionic compounds that contains two cyanine-like segment, which is the expected trend. 30

\section{Results and discussion}

\subsection{Impact of side phenyl rings}

As the computations of the ES vibrations are extremely demanding, we first assess the impact of the side phenyl rings bonded to the nitrogen atoms (see Figure 1). Indeed, experimentally these rings are almost perfectly orthogonal to the core of the dye due to the steric repulsion with the vicinal hydrogen atoms. ${ }^{18}$ We therefore reasoned that there is a strong decoupling between the $\pi$ systems of these phenyl rings and of the core of the compounds. Consequently, the impact of these rings on the optical properties should be minimal. To validate this hypothesis, we first consider 
the lowest $\left(S_{0} \rightarrow S_{1}\right)$ transitions in two representative compounds, namely, 2 and 3. This $S_{0} \rightarrow S_{1}$ transition can be assigned to a dominant HOMO to LUMO electronic promotion (vide infra). As can be seen in Table 1 , the topologies of the frontier orbitals are unaffected by the side phenyl rings, which do not contribute at all to these MOs.

Table 1 Representation of the PCM-PBE0/6-31+G(d) HOMO and LUMO of $\mathbf{2}$ and $\mathbf{3}$ presenting or not $\mathrm{N}$-attached phenyl rings. A contour threshold of 0.02 au has been applied.

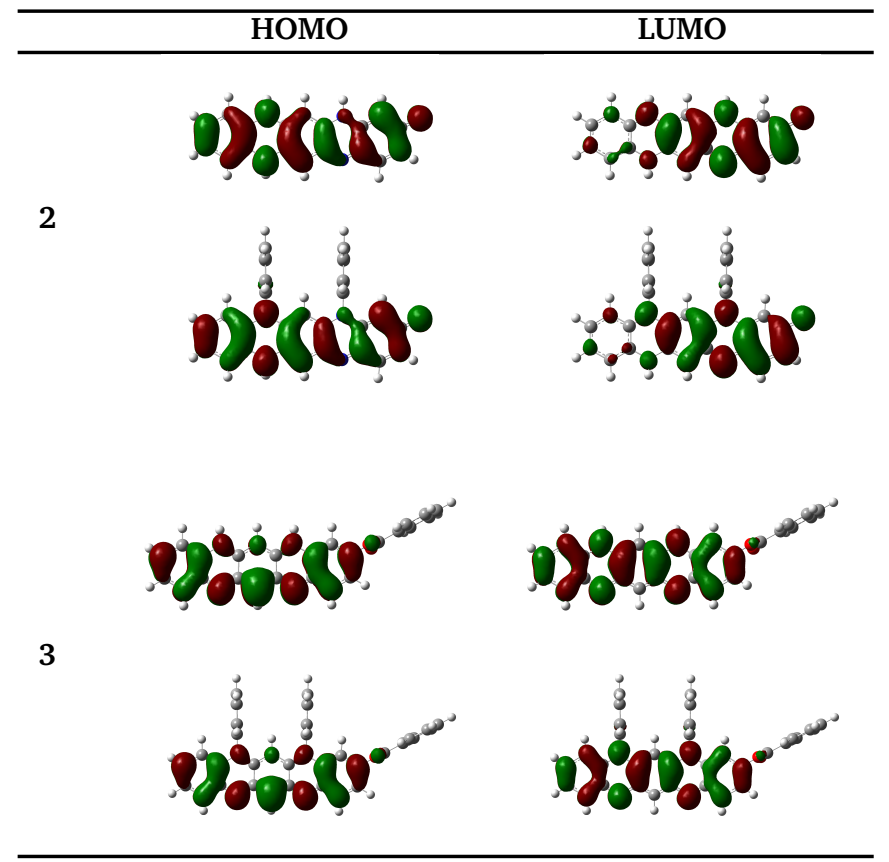

The vibrationally resolved absorption spectra for these two dyes are shown in Figure 2. As can be seen, the band shapes are not affected by the replacement of the phenyl rings by hydrogen atoms, the vibronic progressions being perfectly preserved in both cases. As further detailed in the following of this work, one can clearly note an excellent agreement between the computed and measured band shapes and band positions. One notices that the phenyl rings induce a slight bathochromic displacement of -0.08 $\mathrm{eV}(+29 \mathrm{~nm})$ in 2 and $-0.09 \mathrm{eV}(+44 \mathrm{~nm})$ in 3.50 As these shifts are almost perfectly equal (on the energy scale) for the two systems that present different electronic natures, see Figure 1), we perform all calculations in the following on molecules in which the $\mathrm{N}$-attached phenyl groups have been substituted by hydrogen atoms for the sake of saving computational time.
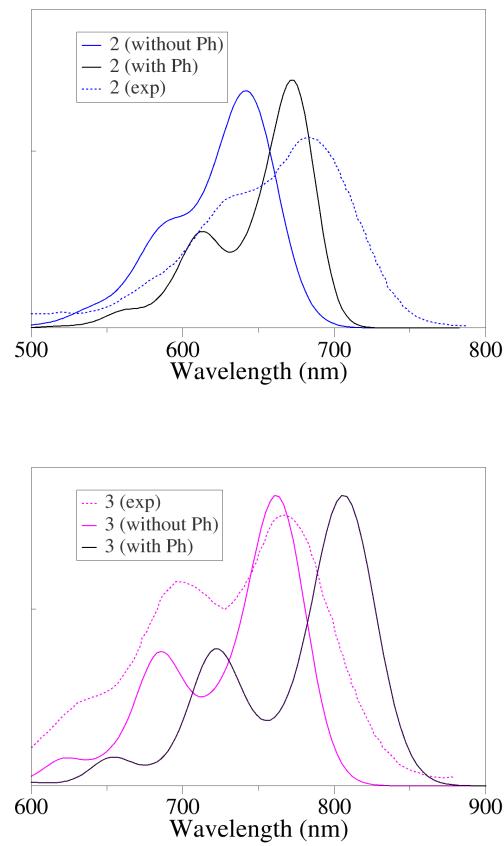

Fig. 2 Impact on the vibrationally-resolved spectra of the $S_{0} \rightarrow S_{1}$ transition of the presence of phenyl rings in 2 (left) and $\mathbf{3}$ (right). The experimental spectra are adapted from Ref. 18

\subsection{Structures}

Before presenting the results of the computations of the optical properties, we briefly discuss the geometrical structures of selected compounds (all GS Cartesian coordinates can be found in the Supplementary Materials). The point groups of the optimal ground-state structures are $\mathrm{C}_{2 v}$ for $\mathbf{1}, \mathrm{C}_{s}$ for 2,5 , and 6 , and $\mathrm{C}_{1}$ for 3 and 4 (due to the side $\mathrm{OBz}$ group), all returning no imaginary frequency. At the exception of $\mathbf{5}$ that becomes $\mathrm{C}_{1}$ in its lowest excited state, all molecules conserve their point group in the $S_{1}$. For compounds 1-4 and 6, that do not change symmetry upon transition, the maximal absolute deviation in bond lengths between the $S_{0}$ and $S_{1}$ states is as small as $0.042 \AA$, a value highlighting the rigidity of the compounds.

In Figure 3, we report the bond lengths determined for both $S_{0}$ and $S_{1}$ in the central section of 1 and 3 . Despite the presence of lateral quinoidal moieties, the central ring of 1 presents an almost phenyl-like structure with a small bond length alternation (BLA $=0.039 \AA$ ) in the GS. When going to $S_{1}$, the changes of bond distances are relatively small (consistent with the abovementioned rigidity) but one notes small contractions of all $\mathrm{CN}$ bonds together with a small elongation of the CC bond connecting the top and bottom moieties of the dye in Figure 3 . In 3, these central bonds are significantly longer than in 1 in $S_{0}(+0.030$ $\AA$ ), leading to a larger BLA of $0.065 \AA$. This change is consistent with the well-known coupling principle $\mathrm{e}^{51}$, that states that the central bond acquires a (partial) single-bond character in such compounds composed of two separated subsystems. This presence of elongated central CC distance has indeed been reported in several zwitterions systems experimentally 5253 . In the lowest ES this central bond tends to contract in 3, whereas changes of opposite signs are found for the $\mathrm{CN}$ bonds of the positively and negatively charged cyanines, suggesting a charge-transfer transition. This highlights the qualitative differences between the neu- 
tral and zwitterionic compounds, that are rationalized in the next Section.

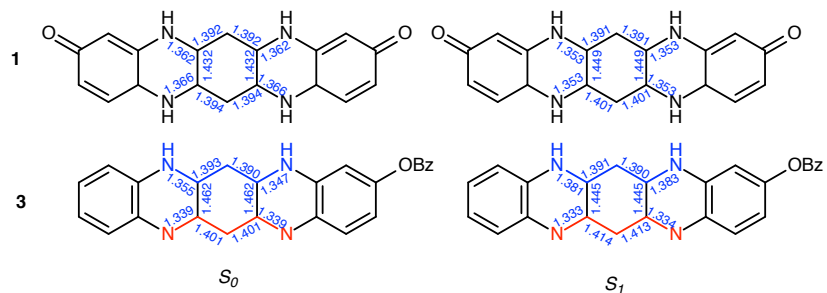

Fig. 3 Bond lengths $(\AA)$ of molecules 1 and 3 in their ground (left) and lowest excited (right) states. All data at the PCM-PBE0/6-31+G(d) level of theory.

\subsection{Nature of the key electronic transitions}

For all six compounds, we provide in Section S.3 of the Supplementary Materials a list of vertical transition energies, oscillator strengths, and major orbital contributions for the ten lowest ES. Although we recall again that such data do not have directlymeasurable equivalents, they provide insights into the nature of the underlying electronic phenomena. For all molecules, the transition with the longest wavelength, corresponding to the measured $\lambda_{\max }$ located in the red region of the spectra, can be associated to a $S_{0} \rightarrow S_{1}$ transition that is strongly dipole allowed ( $f>0.5$ in all six compounds), itself presenting a strongly dominant HOMO-LUMO character. These orbitals can be found in the Supplementary Materials and it can be seen that they present a typical $\pi$ (or $\pi^{\star}$ ) character and are generally highly-delocalized over the full structure as expected for such conjugated chromophores. In all compounds, one finds another strongly dipoleallowed electronic transition in the vertical spectra, e.g., $S_{0} \rightarrow S_{6}$ $(f=0.69)$ in $1, S_{0} \rightarrow S_{5}(f=0.58)$ in $2, S_{0} \rightarrow S_{4}(f=1.19)$ in $\mathbf{3}$, etc. and these transition have also been considered in modeling the actual UV/Vis spectra (see next Section).

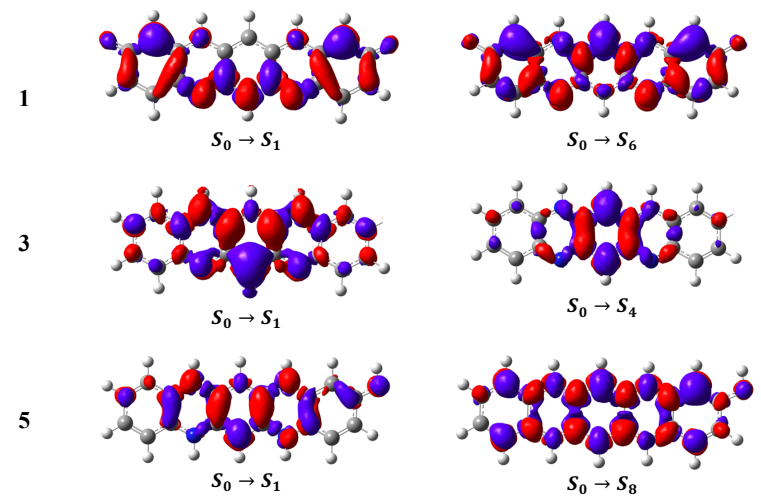

Fig. 4 PCM-PBE0/6-31+G(d) EDD plots for two key electronic transitions (large $f$ ) in $\mathbf{1}, \mathbf{3}$, and $\mathbf{5}$. Blue and red colors indicate density depletion and accumulation, respectively. A contour threshold of $8 \times 10^{-4}$ au has been applied. For the sake of easing the comparison, the OBz group, not taking part in the electronic transitions, is not shown in $\mathbf{3}$.

In Figure 4 we show electronic density difference (EDD) plots for three representative systems. Such plots allow to "show" the impact of the electronic transition more directly than MO plots. In the neutral 1 , going from $S_{0}$ to $S_{1}$ induces a density redistribution towards the bottom part of the molecule (that gains density and appears mostly in red in Figure (4). We note that the central CC bonds appear in blue indicating a decrease of density, consistent with their elongation after excitation (see Figure 3). Interestingly, the next strongly dipole-allowed transition, corresponding to the $S_{6}$ state shows a quite similar pattern, the density moving from the top to the bottom region of the dye. When going to the zwitterionic 3 , there is a strong difference of topology of the ES. Indeed, the $S_{0} \rightarrow S_{1}$ excitation corresponds to a CT from the negatively charged cyanine (bottom, in blue) to the the positively charged one (top, in red), which is typical of such zwitterions build upon two cyanines. $\frac{53154}{5 n}$ One notes a strong gain of density on the central CC bonds, again consistent with the contraction of these bonds found in Figure 3 For the $S_{0} \rightarrow S_{4}$ transition in 3, the changes are localized on the central ring with, again, a strong increase of density on the two CC single bonds. It is also important to note that the dipole moment decreases significantly when going from $S_{0}$ to $S_{1}$ in $3(-2.2 \mathrm{D}$ whereas all other compounds show typical variations of ca. 1 D only), meaning that a negative solvatochromism is expected. Such rather unusual behavior was observed previously in compact zwitterions. .5354 In $\mathbf{5}$, the topology of the first transition is relatively similar to the one of 3 , but with a smaller CT character, the logical consequence of the presence of two positively charged cyanines. Finally, the second bright transition in $\mathbf{5}\left(S_{0} \rightarrow S_{8}\right)$ presents a classical $\pi \rightarrow \pi^{\star}$ nature with many nodes, the state being delocalized over the full system.

\subsection{Analysis of the vibrationally-resolved spectra}

In Table 2 , we provide a comparison between: i) the measured $\lambda$ and $\varepsilon$ for the major bands in the UV/Vis spectra taken from Ref. 18, and ii) the theoretically predicted positions and intensities of the main peaks in the vibrationally-resolved spectra obtained for these main electronic transitions. For the most redshifted band, the mean absolute difference between experiment and theory is $0.10 \mathrm{eV}(33 \mathrm{~nm})$, a small value for such calculations. ${ }^{45 / 47 / 55}$ In addition, the predicted theoretical values are systematically blueshifted, a logical consequence of the removal of the phenyl rings (vide supra). The $R^{2}$, measuring the correlation between the measured and predicted $\lambda_{\max }$, is also excellent (>0.99). For the $\varepsilon$, which are much more challenging to estimate theoretically, $\underline{56}$ the agreement is acceptable, but for 4 , for which theory significantly undershoots the experimental value. For the second bands related to a higher ES, the errors become unsurprisingly slightly larger (mean absolute deviation of $0.17 \mathrm{eV}$ ) but remain within the typical error bar of the selected level of theory.

Table 2 Measured and computed wavelengths of maximal absorption and molar absorption coefficients for dyes 1-6. All computations account for vibronic coupling. The experimental values are taken from Ref. 18

\begin{tabular}{ccc}
\hline & $\begin{array}{c}\text { Experiment } \\
\lambda(\mathrm{nm})[\log \varepsilon]\end{array}$ & $\lambda(\mathrm{nm})[\log \varepsilon]$ \\
\hline $\mathbf{1}$ & $379[4.54]-613[4.81]$ & $366[4.84]-558[5.35]$ \\
$\mathbf{2}$ & $375[4.14]-683[4.47]$ & $413[3.40]-642[4.93]$ \\
$\mathbf{3}$ & $424[4.77]-775[4.50]$ & $476[5.31]-761[4.93]$ \\
$\mathbf{4}$ & $426[4.83]-791[4.43]$ & $444[5.20]-779[3.10]$ \\
$\mathbf{5}$ & $665[4.36]$ & $305[4.58]-626[5.13]$ \\
$\mathbf{6}$ & $385[4.10]-608[4.56]$ & $385[4.76]-569[4.39]$ \\
\hline
\end{tabular}

Figure 5 provides a direct comparison between the measured and calculated spectra for dyes 1-6. Larger representations can 
be found in the Supplementary Materials. It is important to recall that the theoretical spectra are not scaled nor corrected as they are "out-of-the box" results. Obviously, the global agreement between the measurements and the calculations in terms of band positions, widths, heights, and topologies is quite excellent, and the same holds for the relative evolution from one compound to another. This result is inline with a recent work devoted other neutral azaacenes, in which the Dreuw group also recently reported vibrationally resolved theoretical spectra matching experiment. $\frac{57}{57}$ For the presently studied dyes, going from the neutral 1 to the zwitterionic 3 induces a large redshift of the measured $\lambda_{\max }$ by $162 \mathrm{~nm}$ accompanied by a decrease of the $\varepsilon$ by $-17 \%$. The applied theoretical protocol reproduces these trends with respective values of $+203 \mathrm{~nm}$ and $-8 \%$. The second band between 300 and $400 \mathrm{~nm}$ in 1 is due to a $S_{0} \rightarrow S_{6}$ transition and has an intensity about half the one of the main band. In 3 the emergence of a new tight absorption above $400 \mathrm{~nm}$ is reproduced by theory and ascribed to a $S_{0} \rightarrow S_{4}$ excitation. Interestingly the presence of a small band at ca. $475 \mathrm{~nm}$ in the experimental spectrum can be attributed to $S_{0} \rightarrow S_{2}$ transition in this dye. In the dicationic $\mathbf{5}$, both experiment and theory detect a single strong electronic absorption in the visible domain, in between the one of $\mathbf{1}$ and 3; the second significant absorption appearing at $305 \mathrm{~nm}$, according to our calculations, is related to a high-lying $\left(S_{8}\right)$ state.
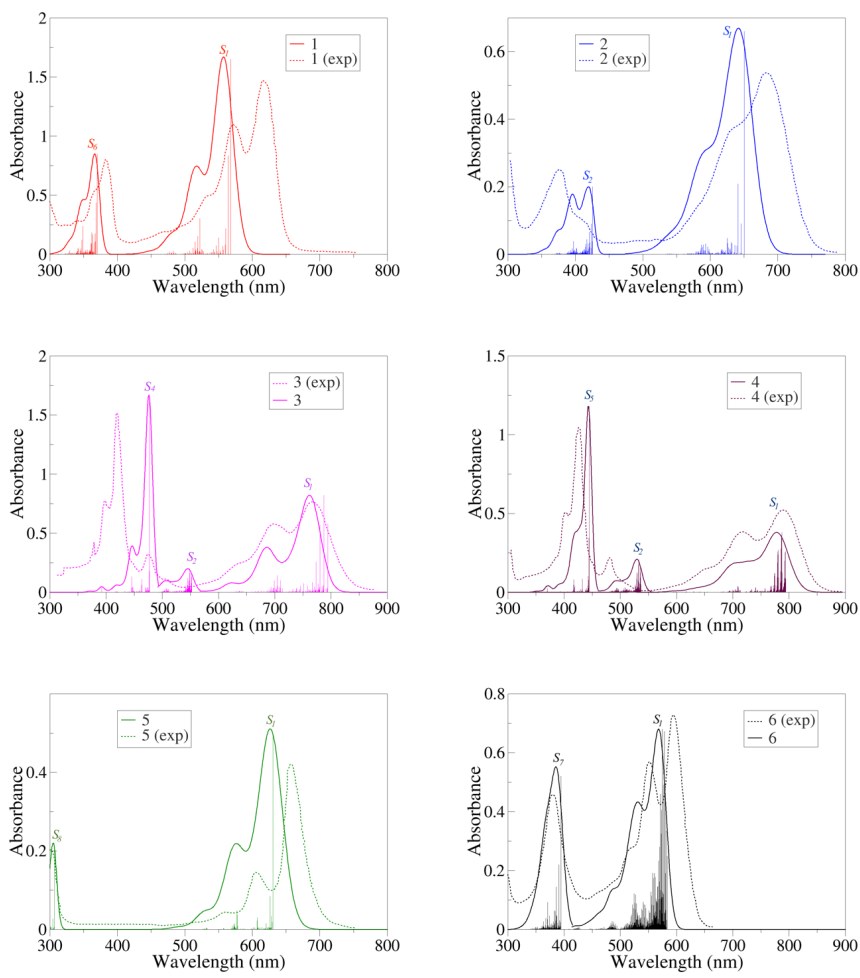

Fig. 5 Comparison between theoretical (full lines and stick contributions) and experimental (dashed lines) UV/Vis absorption spectra for compounds 1-6. The experimental spectra are adapted from Ref. 18 The $0-0$ bands are indicated with numbering of the electronic ES.

In several cases, the vibronic stick contributions displayed in Figure 5 are quite simple, implying that a limited number of vibrational modes are at play to create the multi-peak structures of the electronic absorptions. For instance, the second peak of the main 500-600 $\mathrm{nm}$ band of 1 can be mainly ascribed to an ES vibration taking place at $1438 \mathrm{~cm}^{-1}$. As can be seen in the movie in the Supplementary Materials, this vibration is a stretching mode strongly affecting the CC central and CN bonds with opposite phases for these two families of bonds, a picture completely consistent with the change of geometries reported in Figure 3. For the $S_{0} \rightarrow S_{1}$ transition in 3, a similar mode at 1494 $\mathrm{cm}^{-1}$ explains the presence of the second maxima at ca. 700 $\mathrm{nm}$. However, this mode now presents opposite phases for the extension/contraction of the top and bottom $\mathrm{CN}$ bonds, again consistent with the structures of Figure 3. Although these vibronic calculations have been performed on a simplified version of the dye (see above), the global nature of this key vibration is unaffected by this simplification, which is confirmed by the similar band shapes obtained for phenyl-containing compound (see Figure 2). The second peak in the tight and intense absorption band of 3 (at $400-500 \mathrm{~nm}$ ) is mainly related to two vibrations at 1366 and $1416 \mathrm{~cm}^{-1}$. Finally, in 5, the second peak/shoulder is due to three close-lying vibrations at 1458,1465 , and $1475 \mathrm{~cm}^{-1}$, that are all related to CC/CN stretches but also involve significant wagging contributions from the peripheral hydrogen atoms. Overall, the key vibrations responsible for the specific band shapes of these azaacenes are mainly stretching taking place in the core of the dyes, so that one can expect quite similar band shapes in all substituted compounds as long as the nature of the chromophores is not modified.

\subsection{Design of new compounds}

Given the agreement between experiment and theory for known systems, we decided to apply the same theoretical approach to predict the spectral properties of new (unknown) compounds. We evaluate two strategies here: i) an extension of the $\pi$-conjugated systems; ii) the addition of side donor/acceptor groups that we have applied using $\mathbf{3}$ and $\mathbf{4}$ as references, the latter being inspired by a previous report. $[58$ Substitution has also been showed before to affect gaps in similar systems. ${ }^{[59}$ As above, the interested reader may find in the Supplementary Materials a list of vertical transitions, as well as representation of the key MO, EDD, and vibrationally-resolved spectra. It is noteworthy that in all the new dyes the $S_{0} \rightarrow S_{1}$ transition remains extremely bright whereas a second intense band is always predicted for the $S_{0} \rightarrow S_{3}$ or the $S_{0} \rightarrow S_{4}$ transition.

The two longer systems considered here are displayed in Figure 6 and present an extension by one (7) or two (8) extra phenyl rings. The computed $\lambda$ and $\varepsilon$ for the key transitions are listed in Table 3 whereas Figure 7 provides a comparison between the computed absorption spectra of these two compounds and the one of the parent 3. As can be seen in the EDDs given in the Supplementary Materials, the nature of the $S_{0} \rightarrow S_{1}$ transition in both $\mathbf{7}$ and $\mathbf{8}$ is unchanged as compared to $\mathbf{3}$, i.e., there is a CT from the negatively-charged cyanine to its positively-charged counterpart, the variations of density being strongly localized on the three central rings of the dyes. Despite this outcome, one notices nonnegligible successive redshifts of $+51 \mathrm{~nm}(-0.10 \mathrm{eV})$, and $+70 \mathrm{~nm}$ $(-0.12 \mathrm{eV})$ in the $\mathbf{3} \rightarrow \mathbf{7} \rightarrow \mathbf{8}$ series. In the same time, both the shapes and intensities of this long wavelength band undergo only triffling variations (Figure 7). Other interesting changes are, on the one hand, the significant increase in intensity of the $S_{0} \rightarrow S_{2}$ band in both $\mathbf{7}$ and $\mathbf{8}$ as compared to $\mathbf{3}$, and, on the other hand, the marked blueshift of the tight absorption band at ca. $400 \mathrm{~nm}$ when extending the size of the system.

For evaluating the impact of the addition of electroactive sub- 

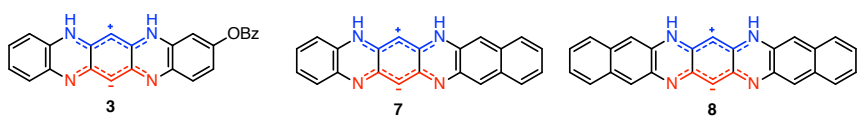

Fig. 6 Molecular structures of $\mathbf{3}, \mathbf{7}$, and $\mathbf{8}$.

Table 3 Predicted wavelength of maximal absorption obtained for dyes 7-16. The results obtained for $\mathbf{3}$ and $\mathbf{4}$ are shown for comparison.

\begin{tabular}{cc}
\hline \multicolumn{1}{c}{ Theory } \\
\multicolumn{1}{c}{$(\mathrm{nm})[\log \varepsilon]$} \\
\hline $\mathbf{3}$ & $476[5.31]-545[2.80]-761[4.93]$ \\
$\mathbf{4}$ & $444[5.20]-530[2.30]-779[3.10]$ \\
$\mathbf{7}$ & $404[4.92]-497[4.43]-812[4.95]$ \\
$\mathbf{8}$ & $415[5.37]-512[4.87]-882[5.01]$ \\
$\mathbf{9}$ & $402[5.19]-479[4.04]-729[5.05]$ \\
$\mathbf{1 0}$ & $399[5.27]-478[5.17]-773[4.86]$ \\
$\mathbf{1 1}$ & $391[5.39]-454[3.79]-802[4.88]$ \\
$\mathbf{1 2}$ & $385[5.26]-508[4.56]-724[5.01]$ \\
$\mathbf{1 3}$ & $398[5.13]-498[4.16]-721[5.19]$ \\
$\mathbf{1 4}$ & $399[5.10]-484[4.16]-812[4.85]$ \\
$\mathbf{1 5}$ & $350[5.39]-448[3.79]-862[4.88]$ \\
$\mathbf{1 6}$ & $398[5.20]-523[4.95]-698[5.13]$ \\
\hline
\end{tabular}

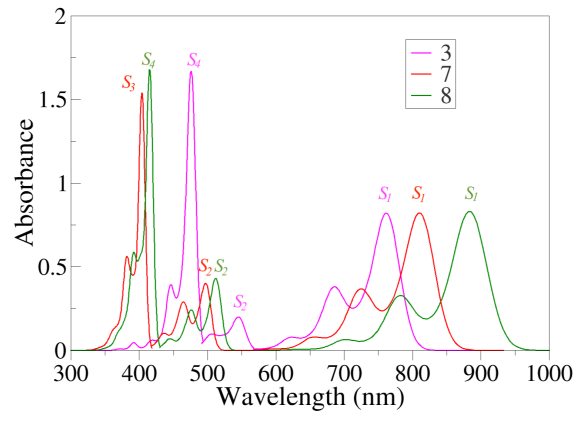

Fig. 7 Comparison between theoretical absorption spectra of $\mathbf{3}, \mathbf{7}$, and 8. Stick spectra can be found in the Supplementary Materials. stituents, we select the amino and cyano side groups as examples of strong donor and acceptor moieties, respectively. The considered compounds are displayed in Figure 8 and show both mono- (9-12) and di- (13-16) substituted compounds, the active group(s) being located in a para configuration compared either to the negatively-charged segment (odd-numbered compounds, center in Figure 8) or its positive counterpart (even-numbered compounds, r.h.s. in Figure 8). The obtained wavelengths are listed in Table 3, and representative EDD and computed spectra can be found in Figures 9 and 10 respectively.

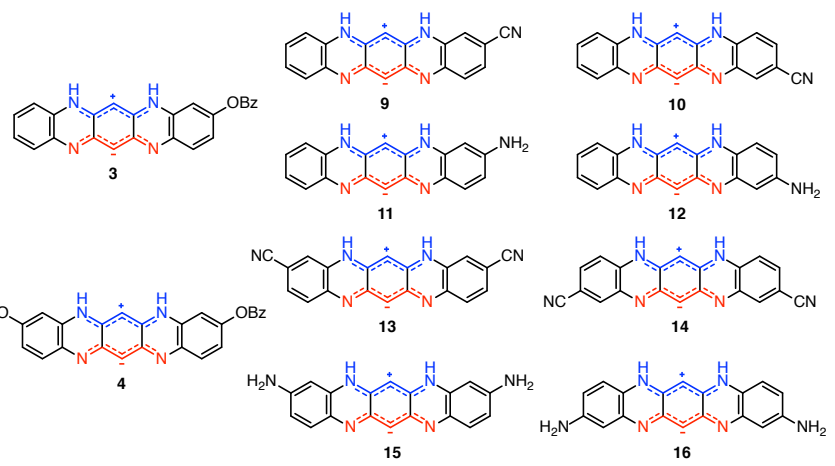

Fig. 8 Molecular structures of compounds 3, 4, and 9-16.

First, we note that the relative evolutions of the mono- and disubstituted compounds, as respectively compared to $\mathbf{3}$ and $\mathbf{4}$, are identical, the double substitution logically inducing quantitatively larger effects. We therefore focus on 13-16 in the following, the conclusions being transposable to 9-12, at the very least for the first electronic transition. One notices in Figure 9 that the nature of the lowest transition is essentially unchanged by the substitution, so that substituents act as actual auxochromes and do not yield to the emergence of a new chromophore. When going from 4 to 13 , a significant blueshift of the $\lambda_{\max }$ by $-58 \mathrm{~nm}(+0.13 \mathrm{eV})$ is predicted by theory. In 13 , one adds electron acceptor groups para of the negative charge located on the "bottom" cyanine segment in the GS (Figure 8). This region acts as the donor moiety in the $S_{0} \rightarrow S_{1}$ CT transition (blue lobes in Figure 9). Consequently, the cyano groups relatively stabilize more $S_{0}$ than $S_{1}$, the CT becomes more difficult in $\mathbf{1 3}$ than in $\mathbf{4}$, and the associated transition energy increases. In contrast, in $\mathbf{1 4}$ the two cyano segments are located ideally to stabilize the electronic density added to the positive segment after the CT (red regions in Figure 9) and hence a redshift of the absorption band is logically crystal-balled by theory $(+33 \mathrm{~nm}$ or $-0.06 \mathrm{eV})$. When using amino donor groups, the effects become, as expected, reversed. Indeed, a strong redshift $(+83 \mathrm{~nm}$ or $-0.15 \mathrm{eV}$ ) is predicted when going from 4 to 15 , this latter dye being predicted to absorb at $862 \mathrm{~nm}$, a large value given the relatively small size of this dye. In 15, the amino groups act as auxiliary donors (in blue in Figure 9) facilitating the CT by destabilizing the negative charge in the GS. In 16, the computational protocol returns a blueshift of $-81 \mathrm{~nm}(+0.19 \mathrm{eV})$ compared to 4, explainable using the same reasoning: the amino groups stabilize the positive charge in the GS, which makes the CT harder to achieve. The impact of the amino groups on the $\lambda_{\max }$ is quantitatively larger than the one of cyano groups, and this effect is typical of cyanine and related derivatives, e.g. BODIPY, that are more affected by donor than acceptor groups. ${ }^{6061}$ As can be seen in Table 3 and Figure 10 , irrespective of the substitution pattern in the 9-16 series, the $S_{0} \rightarrow S_{2}$ transition becomes significantly 
more intense and is blueshifted as compared to 3 and 4 , whereas the high-intensity absorption around $400 \mathrm{~nm}$ also undergoes a blueshift, an effect particularly marked in $\mathbf{1 5}$.
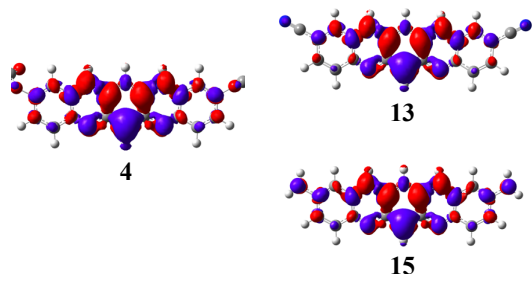
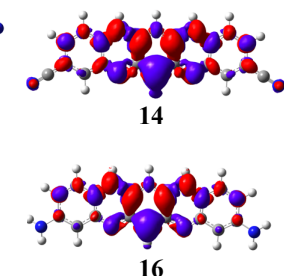

Fig. 9 PCM-PBE0/6-31+G(d) EDD plots for the $S_{0} \rightarrow S_{1}$ transition in 4 and 13-16. For the sake of easing the comparison, the OBz groups, not taking part in the transition, are not shown in 4 . See caption of Figure 4 for more details.
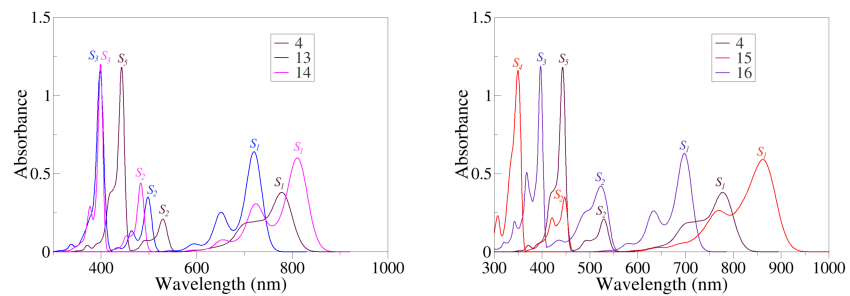

Fig. 10 Comparison between theoretical absorption spectra of 4 and 13-16. Stick spectra can be found in the Supplementary Materials.

\section{Conclusions and outlook}

To the best of our knowledge, this work stands as the first theoretical investigation of the optical signatures of linear azaacenes of various nature (neutral, zwitterionic, and dicationic) using an approach accounting for vibronic effects for all relevant electronic transitions. In more details, the used protocol relies on adiabatic energies determined with the second-order coupled-cluster method, that is well adequate for many classes of dyes, including the one encompassing cyanine-like segments, whereas the vibronic couplings are obtained using the adiabatic Hessian model on the basis of vibrational frequencies analytically determined at the Time-Dependent Density Functional Theory level. Such an approach is able to reproduce with a very good to excellent accuracy the key spectral features of the investigated compounds: positions and shapes of all absorption bands, relative intensities of the peaks, auxochromic effects, etc. A combined analysis of the structural changes upon electronic transition, density difference plots, and vibronic couplings provides a coherent description of the nature of the electronic transitions of the various compounds. In particular, in the zwitterionic derivatives, the $\lambda_{\max }$ absorption corresponds to a charge-transfer transition from the negativelycharged segment to its positively-charged counterpart, this transition being strongly coupled to a vibrational mode modifying the CC and CN bonds lengths in the core of the dye. New derivatives are also discussed and their spectral evolution rationalized. To redshift the absorption band, one can either: i) extend the $\pi$-system, as going from five to seven fused six-member rings induces a large bathochromic shift of $+121 \mathrm{~nm}$, or ii) add amino substituents para of the negatively charged moiety to destabilize it which indues a redshift of $+82 \mathrm{~nm}$ according to our calculations.
We do hope that this work will stimulate new synthesis and experimental characterizations in the field. We are currently pursuing our efforts to model unconventional zwitterionic systems even more accurately.

\section{Conflicts of interest}

There are no conflicts to declare.

\section{Acknowledgements}

This research used resources of: 1) the GENCI-CINES/IDRIS, 2) CCIPL (Centre de Calcul Intensif des Pays de Loire) and 3) a local Troy cluster.

\section{Notes and references}

1 Organic Electronics: Materials, Manufacturing and Applications, ed. H. Klauk, WILEY-VCH Verlag GmbH, 2006.

2 Physical and Chemical Aspects of Organic Electronics: From Fundamentals to Functioning Devices, ed. C. Wöll, Wiley-VCH, 2009.

3 M. Winkler and K. N. Houk, J. Am. Chem. Soc., 2007, 129, 1805-1815.

4 S.-Z. Weng, P. Shukla, M.-Y. Kuo, Y.-C. Chang, H.-S. Sheu, I. Chao and Y.-T. Tao, ACS App. Mat. Int., 2009, 1, 2071-2079.

5 Z. Liang, Q. Tang, J. Liu, J. Li, F. Yan and Q. Miao, Chem. Mater., 2010, 22, 6438-6443.

6 Y.-Y. Liu, C.-L. Song, W.-J. Zeng, K.-G. Zhou, Z.-F. Shi, C.-B. Ma, F. Yang, H.-L. Zhang and X. Gong, J. Am. Chem. Soc., 2010, 132 16349-16351.

7 U. H. F. Bunz, J. U. Engelhart, B. D. Lindner and M. Schaffroth, Angew. Chem. Int. Ed., 2013, 52, 3810-3821.

8 T. Lelaidier, T. Leoni, P. Arumugam, A. Ranguis, C. Becker and O. Siri, Langmuir, 2014, 30, 5700-5704.

9 B. Kollmann, Z. Chen, D. Lüftner, O. Siri and P. Puschnig, J. Phys. Chem. C, 2018, 122, 6475-6482.

10 A. Thomas, W. Malone, T. Leoni, A. Ranguis, Z. Chen, O. Siri, A. Kara, P. Zeppenfeld and C. Becker, J. Phys. Chem. C, 2018, 122, 1082810834.

11 Q. Miao, Adv. Mater., 2014, 26, 5541-5549.

12 U. H. Bunz, Acc. Chem. Res, 2015, 48, 1676-1686.

13 J. Li and Q. Zhang, ACS. Appli. Surf Int, 2015, 7, 28049-28062.

14 P.-Y. Gu, Z. Wang and Q. Zhang, J. Mater. Chem B, 2016, 4, 70607074.

15 M. Ganschow, S. Koser, S. Hahn, F. Rominger, J. Freudenberg and U. H. Bunz, Chem. Eur. J., 2017, 23, 4415-4421.

16 H. Pan, T. Song, X. Yin, P. Jin and J. Xiao, Chem. Eur. J., 2018, 24, $6572-6579$.

17 G. A. Zissimou, C. P. Constantinides, M. Manoli, G. K. Pieridou, S. C. Hayes and P. A. Koutentis, Org. Lett, 2016, 18, 1116-1119.

18 G. A. Zissimou, A. Kourtellaris and P. A. Koutentis, J. Org. Chem., 2018, 83, 4754-4761.

19 G. A. Zissimou, A. Kourtellaris and P. A. Koutentis, Org. Lett, 2018, 20, 844-847.

20 M. Dierksen and S. Grimme, J. Phys. Chem. A, 2004, 108, 1022510237.

21 F. Santoro, A. Lami, R. Improta, J. Bloino and V. Barone, J. Chem. Phys., 2008, 128, 224311.

22 D. Jacquemin, A. Planchat, C. Adamo and B. Mennucci, J. Chem. Theory Comput., 2012, 8, 2359-2372.

23 F. J. Avila Ferrer, J. Cerezo, E. Stendardo, R. Improta and F. Santoro, J. Chem. Theory Comput., 2013, 9, 2072-2082.

24 A. Charaf-Eddin, A. Planchat, B. Mennucci, C. Adamo and D. Jacquemin, J. Chem. Theory Comput., 2013, 9, 2749-2760.

25 F. Muniz-Miranda, A. Pedone, G. Battistelli, M. Montalti, J. Bloino and V. Barone, J. Chem. Theory Comput., 2015, 11, 5371-5384.

26 F. Vazart, C. Latouche, J. Bloino and V. Barone, Inorg. Chem., 2015, 54, 5588-5595.

27 S. Di Tommaso, D. Bousquet, D. Moulin, F. Baltenneck, P. Riva, H. David, A. Fadli, J. Gomar, I. Ciofini and C. Adamo, J. Comput. Chem., 2017, 38, 998-1004.

28 M. Schreiber, V. Bub and M. P. Fülscher, Phys. Chem. Chem. Phys., 2001, 3, 3906-3912. 
29 S. Grimme and F. Neese, J. Chem. Phys., 2007, 127, 154116.

30 B. Le Guennic and D. Jacquemin, Acc. Chem. Res., 2015, 48, 530-537.

31 S. Chibani, A. D. Laurent, B. Le Guennic and D. Jacquemin, J. Chem. Theory Comput., 2014, 10, 4574-4582.

32 M. R. Momeni and A. Brown, J. Chem. Theory Comput., 2015, 11, 2619-2632.

33 M. J. Frisch, G. W. Trucks, H. B. Schlegel, G. E. Scuseria, M. A. Robb, J. R. Cheeseman, G. Scalmani, V. Barone, G. A. Petersson, H. Nakatsuji, X. Li, M. Caricato, A. V. Marenich, J. Bloino, B. G. Janesko, R. Gomperts, B. Mennucci, H. P. Hratchian, J. V. Ortiz, A. F. Izmaylov, J. L. Sonnenberg, D. Williams-Young, F. Ding, F. Lipparini, F. Egidi, J. Goings, B. Peng, A. Petrone, T. Henderson, D. Ranasinghe, V. G. Zakrzewski, J. Gao, N. Rega, G. Zheng, W. Liang, M. Hada, M. Ehara, K. Toyota, R. Fukuda, J. Hasegawa, M. Ishida, T. Nakajima, Y. Honda, O. Kitao, H. Nakai, T. Vreven, K. Throssell, J. A. Montgomery, Jr., J. E. Peralta, F. Ogliaro, M. J. Bearpark, J. J. Heyd, E. N. Brothers, K. N. Kudin, V. N. Staroverov, T. A. Keith, R. Kobayashi, J. Normand, K. Raghavachari, A. P. Rendell, J. C. Burant, S. S. Iyengar, J. Tomasi, M. Cossi, J. M. Millam, M. Klene, C. Adamo, R. Cammi, J. W. Ochterski, R. L. Martin, K. Morokuma, O. Farkas, J. B. Foresman and D. J. Fox, Gaussian 16 Revision A.03, 2016, Gaussian Inc. Wallingford CT.

34 C. Adamo and V. Barone, J. Chem. Phys., 1999, 110, 6158-6170.

35 M. Ernzerhof and G. E. Scuseria, J. Chem. Phys., 1999, 110, 50295036.

36 A. D. Laurent and D. Jacquemin, Int. J. Quantum Chem., 2013, 113, 2019-2039.

37 J. Tomasi, B. Mennucci and R. Cammi, Chem. Rev., 2005, 105, 29993094.

38 C. A. Guido and S. Caprasecca, Int. J. Quantum Chem., 2019, 119, e25711.

39 R. Cammi and B. Mennucci, J. Chem. Phys., 1999, 110, 9877-9886.

40 M. Cossi and V. Barone, J. Chem. Phys., 2001, 115, 4708-4717.

41 M. Caricato, B. Mennucci, J. Tomasi, F. Ingrosso, R. Cammi, S. Corni and G. Scalmani, J. Chem. Phys., 2006, 124, 124520.

42 F. J. Avila Ferrer and F. Santoro, Phys. Chem. Chem. Phys., 2012, 14, 13549-13563.

43 F. Santoro and D. Jacquemin, WIREs Comput. Mol. Sci., 2016, 6, 460486.

44 F. Santoro, R. Improta, A. Lami, J. Bloino and V. Barone, J. Chem. Phys., 2007, 126, 184102.

45 D. Jacquemin, I. Duchemin and X. Blase, J. Chem. Theory Comput., 2015, 11, 5340-5359.

46 R. Send, M. Kühn and F. Furche, J. Chem. Theory Comput., 2011, 7, 2376-2386.

47 N. O. C. Winter, N. K. Graf, S. Leutwyler and C. Hättig, Phys. Chem. Chem. Phys., 2013, 15, 6623-6630.

48 P.-F. Loos and D. Jacquemin, J. Chem. Theory Comput., 2019, 15, 2481-2491.

49 TURBOMOLE V6.6 2014, a development of University of Karlsruhe and Forschungszentrum Karlsruhe GmbH, 1989-2007, TURBOMOLE GmbH, since 2007; available from http: / / www . turbomole.com (accessed 13 June 2016).

50 For 2, the computed $\lambda_{\max }$ are 671 and $642 \mathrm{~nm}$ with and without the phenyl rings (experimental value: $683 \mathrm{~nm}$ ). For 3 , the computed $\lambda_{\max }$ are 805 and $761 \mathrm{~nm}$ with and without the phenyl rings (experimental value: $775 \mathrm{~nm}$ ).

51 S. Dähne and D. Leupold, Angew. Chem. Int. Ed., 1966, 5, 984-993.

52 Z. Chen, M. Giorgi, D. Jacquemin, M. Elhabiri and O. Siri, Angew. Chem. Int. Ed., 2013, 52, 6250-6254.

53 S. Pascal, L. Lavaud, C. Azarias, G. Canard, M. Giorgi, D. Jacquemin and O. Siri, Mater. Chem. Front., 2018, 2, 1618-1625.

54 S. Pascal, L. Lavaud, C. Azarias, A. Varlot, G. Canard, M. Giorgi, D. Jacquemin and O. Siri, J. Org. Chem., 2019, 84, 1387-1397.

55 L. Goerigk and S. Grimme, J. Chem. Phys., 2010, 132, 184103.

56 A. Charaf-Eddin, T. Cauchy, F.-X. Felpin and D. Jacquemin, RSC Adv., 2014, 4, 55466-55472.

57 M. Hodecker, M. Ganschow, M. Abu-Odeh, U. H. F. Bunz and A. Dreuw, ChemPhotoChem, 2019, 0, 000-000.

58 C. P. Constantinides and P. A. Koutentis, in Effects of substitution on the ground-state multiplicities of zwitterionic polyazaacenes: A DFT study combined with broken symmetry approach, University of Wollongong, 2004.

59 C. P. Constantinides, T. A. Ioannou and P. A. Koutentis, Polyhedron,
2013, 64, 172-180.

60 S. Chibani, B. Le Guennic, A. Charaf-Eddin, A. D. Laurent and D. Jacquemin, Chem. Sci., 2013, 4, 1950-1963.

61 S. Pascal, A. Haefele, C. Monnereau, A. Charaf-Eddin, D. Jacquemin, B. Le Guennic, C. Andraud and O. Maury, J. Phys. Chem. A, 2014, 118, 4038-4047. 
TOC

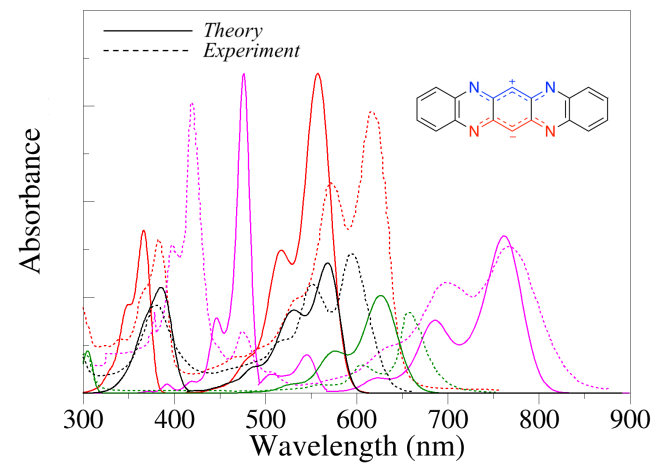

